Instituto Internacional de Investigación y Desarrollo Tecnológico Educativo INDTEC, C.A.

DOI: https://doi.org/10.29394/Scientific.issn.2542-2987.2021.6.21.19.356-375

OAl-PMH: http://www.indteca.com/ojs/index.php/Revista Scientific/oai

Ensayo Original / Original Essay

\title{
Perspectivas de la Formación Académica desde una Visión Trascendental Universitaria
}

\author{
Autora: Maribel Josefina Díaz Castellanos \\ Universidad Nacional Experimental "Rafael María Baralt", UNERMB \\ maribdiazcastellanos@gmail.com \\ Maracaibo, Venezuela \\ https://orcid.org/0000-0002-6766-3277
}

\section{Resumen}

El presente ensayo plasma a manera de reflexión y crítica constructiva un enfoque de la formación académica desde una visión transcendental en el ámbito universitario. En este documento, se desglosan tres aspectos: primero, la formación académica, enmarcada en generar planes de formación que permita la constante actualización, preparación, superación académica como desarrollo intelectual del Profesor Universitario, así como, en vanguardia con el cambiante desarrollo científico, tecnológico y humanístico que engloba la Universidad. Segundo, la formación del personal académico versus realidad universitaria; confronta la contribución hacia el desarrollo, capacitación, adaptabilidad de la amplia formación académica profesional en el rol del cuerpo profesoral universitario comenzando con la significación como formador integral del egresado universitario. Tercero, la visión transformadora universitaria, propicia dentro de las Instituciones de Educación Universitaria, promoviendo una cultura de formación académica imprescindible para resaltar la excelencia, equidad y calidad educativa dentro de las Universidades Venezolanas. Para finalmente, presentar con sentido de pertinencia universitaria, las conclusiones, emitiendo juicios de valor relacionados a la formación profesoral en sus funciones académicas, dirigida hacia la cimentación de acciones viables en concordancia con la visión de la transformación universitaria requerida, basadas en los estudios de Cabero (2007); Escamilla (2012); García (1998); Inciarte y Canquiz (2008); y Yarzábal (1999).

Palabras clave: formación académica; docente; universidad. Código de clasificación internacional: 5802.07 - Formación profesional.

Cómo citar este ensayo:

Díaz, M. (2021). Perspectivas de la Formación Académica desde una Visión Trascendental Universitaria. Revista Scientific, 6(21), 356-375, e-ISSN: 2542-2987. Recuperado de: https://doi.org/10.29394/Scientific.issn.2542-2987.2021.6.21.19.356-375

Fecha de Recepción: 05-04-2021
Fecha de Aceptación: 05-07-2021
Fecha de Publicación: 05-08-2021 


\title{
Perspectives of the Academic Formation from a Transcendental University Vision
}

\begin{abstract}
This essay reflects, as a reflection and constructive criticism, an approach to academic training from a transcendental vision in the university environment. In this document, three aspects are broken down: first, academic training, framed in generating training plans that allow constant updating, preparation, academic improvement and intellectual development of the University Professor, as well as, at the forefront with the changing scientific and technological development and humanistic that encompasses the University. Second, the training of academic staff versus university reality; It confronts the contribution towards the development, training, adaptability of the broad professional academic training in the role of the university faculty, starting with the significance as an integral educator of the university graduate. Third, the university transforming vision, fostered within the University Education Institutions, promoting a culture of academic training essential to highlight educational excellence, equity and quality within Venezuelan Universities. In order to finally present the conclusions with a sense of university relevance, issuing value judgments related to teacher training in their academic functions, directed towards the foundation of viable actions in accordance with the vision of the required university transformation, based on the studies of Cabero (2007); Escamilla (2012); García (1998); Inciarte y Canquiz (2008); and Yarzábal (1999).
\end{abstract}

Keywords: academic training; teacher; college. International classification code: 5802.07 - Vocational training.

How to cite this essay:

Díaz, M. (2021). Perspectives of the Academic Formation from a Transcendental University Vision. Revista Scientific, 6(21), 356-375, e-ISSN: 2542-2987. Recovered from: https://doi.org/10.29394/Scientific.issn.2542-2987.2021.6.21.19.356-375

Date Received: 05-04-2021
Date Acceptance: 05-07-2021
Date Publication: 05-08-2021 


\section{Introducción}

Ante la actual realidad apreciada desde todos los ámbitos en especial particular el Universitario, no escapa a todas las necesidades propias que amerita un cambio de adaptación proclive a generar una educación académica de excelencia, con la garantía de una formación de su personal profesoral cada vez más versátil, además, cónsona con las manifestaciones que en la Venezuela de hoy se requiere y es urgente discutir, dialogar, proyectar; para proponer en aras de una nueva visión universitaria nacional, latinoamericana y mundial.

Por esto, los cambios que se presentan a nivel mundial exigen cada vez más de Instituciones Educativas Universitarias inmersas en un proceso transformador, proactivo que configure el "estatus quo" de la realidad con lo que se ejecuta, por lo que, este ensayo, bordea el factor y sentir del Profesor Universitario desde la Formación Académica con una visión trascendental universitaria.

Del mismo modo, toda la temática permite un análisis crítico, reflexivo y lógico que engloba la Formación Académica; delineando la formación del personal académico universitario para elevar el considerado perfil, actualización, preparación, pericia y habilidades que pretende evolucionar en función al conocimiento, área y ciencia que estudia, investiga, indaga y transmite en el quehacer diario de la vida formativa universitaria.

Continuando con el proceso de interrelacionar el devenir en la visión de la Formación del Personal Académico versus Realidad Universitaria; se sostiene que las Instituciones de Educación Universitaria Venezolanas están en el deber de formar a un personal que genera el desempeño a nivel académico, profesional y científico, lo cual, representa proactividad docente, para así, poder asimilar los cambios producto del desarrollo científico, educacional, tecnológico, humanístico e instruccional, con el fiel propósito de garantizar la constante actualización con los nuevos enfoques, teorías y 
construcción de nuevos elementos filosóficos-epistemológicos que perfilan y condensan de forma íntegra al Profesor Universitario.

Asimismo, el tercer tópico describe los aspectos a plantear desde las Instituciones de Educación Universitaria con la finalidad de incentivar el mejoramiento continuo de su Personal Docente Universitario y los diversos cambios, así como, la puesta en marcha de planes de acción enlazados a las políticas de Estado que pretendan consolidar el logro de la maximización del talento humano profesoral transfiriendo consecuencialmente; calidad, equidad y excelencia académica universitaria.

Por último, el presente escrito aborda las conclusiones para ejercer énfasis en la visión universitaria desde una profunda preocupación por la formación docente universitaria que vislumbre la manera como se realice la introspección en las Instituciones de Educación Universitaria para fortalecer y buscar la vanguardia académica, científica, humanística y social que el contexto demanda.

En este sentido se comprende, que el objetivo pretende proyectar la realidad universitaria desde la formación académica y presentar los retos que se requieren hacia la transformación universitaria.

\section{Desarrollo}

\subsection{Formación Académica}

A nivel de las Instituciones de Educación Universitarias (IEU), los desafíos están direccionados a formar, establecer e instituir que su Planta Profesoral, sean docentes e investigadores científicos, formadores y responsables de la calidad de los procesos de enseñanza y la cualidad de los aprendizajes, pero sobre todas las cosas con congruencia y pertinencia social, por ende, García (1998): manifiesta que deben estar comprometidos con lo significativo de las diferentes profesiones, en cuanto a su crecimiento personal, desarrollo intelectual y el progreso del potencial de su inteligencia individual 
en función del contexto real educativo-económico-socio-político circundante.

En este contexto la Educación Universitaria, las estrategias de enseñanza-aprendizaje requieren la garantía de un profesional que aprende haciendo, aprende a aprender y aprender en colectivo en todos los ámbitos, espacios, ciencias y disciplinas del conocimiento. En consecuencia, el reto del profesor universitario es divulgar un sólido conocimiento de las asignaturas que administra, transmitir sus saberes y transferir competencias investigativas, trabajo multi e interdisciplinario, consolidación del saber y actuar didáctico, es construir a partir de un nuevo sistema de instrucción un profesional muy bien preparado, crítico, ético, responsable, solidario, y que contribuya con el avance, bienestar, evolución, grandeza, prosperidad e impulso de la nación, en aras de favorecer el interés de la construcción del nuevo país.

Con base en lo expuesto, es evidente, indudable e irrefutable que la formación del personal académico universitario debe ser una característica básica, una constante intrínseca e innata a cada profesor, de carácter continuo y de permanente actualización, mejora, acrecentamiento, preparación, perfección de sus estudios, competencias, destrezas, habilidades e instrucción, donde exista reflexión, socialización, producción y construcción de saberes vinculados al contexto y al conocimiento mismo, con la finalidad de contagiar, estimular, motivar, provocar e incrementar en los estudiantes sus niveles de compromisos propios con su formación de Pregrado, en conformidad para elevar e incrementar los horizontes de calidad, condición, y excelencia de la academia universitaria.

Al mismo tiempo, es de vital importancia para el profesor universitario el entender, compartir postura, coincidir en la percepción e internalizar que es posible educar para un mejor mundo, lo cual va a conducir a concebir y emprender el proceso educativo como un fértil terreno para promover, desplegar y favorecer el adelanto, creación e introducción de ambientes educacionales universitarios activos, dinámicos, eficientes, perseverantes con 
la singularidad de una instrucción con formación crítica, en inéditos ambientes de procesos enseñanza - aprendizaje planificados en la diversidad de las estrategias didácticas, con distintos procedimientos instruccionales, reflejados en pensum de: adiestramiento, capacitación, estudios, inmersos en sistemas alternativos de formación para transformar la manera de producir, reproducir, difundir y socializar el conocimiento.

Es importante resaltar la reflexión de Escamilla (2012): donde indica la representación media proporcional del estudiantado: target adultos; que se fijan un propósito de estudio por necesidad, por estatus, para satisfacer un logro personal profesional que casi siempre contrae disyuntivas adversas como el tiempo a invertir, dinero, herramientas tecnológicas que aprender, afrontar paradigmas de usos arcaicos que se han instaurado por facilidad, comodidad o falta de motivación en asumir cambios para mejor.

En este orden de ideas, todo esto se sopesa equilibradamente ante las oportunidades que ofrece el uso de la formación a través del uso de la tecnología educativa con innumerables ventajas como flexibilidad en el tiempo, espacio, la oferta educativa disponible, incremento de la interacción facilitadorparticipantes que promueva a la producción de intercambios de conocimientos para aplicar de manera inmediata en el ámbito laboral o personal.

Dentro de este marco, en las Instituciones de Educación Universitaria venezolana, muchas de ellas aún en la actualidad permanecen aisladas, encerradas, enclaustradas y profesan doctrinas, dogmas donde no se permiten profesores con diferencia de opiniones, ni variedad de puntos de vista, que disientan y no se ajusten subordinándose automáticamente a las políticas-filosofías de la Institución de Educación Universitaria, ni estudiantes críticos que exijan una transformación de la cultura universitaria, los conocimientos, los ámbitos educacional-científico-social-político-económico, los principios e ideales; que para Yarzábal (1998): se encamine, encauce y oriente a abordar, aproximarse, entender no sólo únicamente las realidades 
educativas actuales, sino también de la sociedad en general, que fomente la construcción de un nuevo orden social.

Por otra parte, es importante mencionar según Cabero (2007): que las Instituciones de Educación Universitaria enfrentan retos sin precedentes y encaran las nuevas formas de preparar a los individuos mediante modelos educativos flexibles, accesibles para los necesitados de satisfacer la formación continua en adaptación del talento humano. Las Instituciones de Educación Universitaria en constante adaptación a los cambios con una nueva era de la información se ve obligada a cambiar, a ofrecer una formación integral flexible para adaptarse, apropiarse y habituarse a nuevas situaciones, adquirir nuevos conocimientos siendo capaces de cuestionar viejos paradigmas.

Se observa todo esto, mediante la plataforma tecnológica, catalogado como un espacio virtual que por medios de recursos tecnológicos muestran materiales de un contenido específico que permita la construcción y transferencia de conocimientos entre los miembros de un grupo, logrando la asimilación efectiva del conocimiento.

De esta manera, tomando en consideración lo antes expuesto, las Instituciones de Educación Universitaria deben por el contrario comunicar, inspirar, infundir e inyectar una actitud participativa, mancomunada y disposición solidaria con valores en la comunidad, promoviendo la convivencia con los actores concurrentes e inmersos en el hecho educativo, sectores sociales y factores productivos reales de las comunidades, además de actuar, apoyar, contribuir e intervenir de forma activa, decisoria, eficaz, emprendedora y positiva como una Instituciones de Educación Universitaria Transformadora.

En esta dirección, el esfuerzo de todos los involucrados se orientará principalmente a diseñar, elaborar, desarrollar e instaurar proyectos, programas, perfiles curriculares que desplieguen, extiendan, promuevan y perfeccionen el valor del campo educativo, donde los habitantes, la localidad, los sectores productivos de bienes y servicios, es decir, la población en su 
conjunto se vea comprometida, implicada, envuelta e involucrada en el objetivo de atender, solucionar, solventar y subsanar las carencias, necesidades y problemas reales.

Por ello, se hace necesario que la formación académica de las autoridades de cada Institución de Educación Universitaria y de su cuerpo profesoral los faculten, habiliten para permitir acceder a estudiar, observar e implementar acciones educacionales, prácticas e instruccionales relacionadas con lo concerniente de cómo aprovechar todas las potencialidades de la población para que los planes, propuestas y políticas curriculares propias vayan de la mano en promover la formación, preparación, egreso e incorporación de nuevos profesionales al entorno siendo mano de obra calificada con elevados niveles de: adiestramiento, capacitación, disposición, organización, planificación, saberes.

Igualmente, es pertinente el establecer por parte de las Universidades las asesorías-consultas técnicas de atención a las comunidades, ejecutar programas de administración, cogobierno, gestión y control, participación, movilización, en afinidad, con su novedosa condición de estructura organizacional institucional educativa-social-popular, es decir, la misión y visión de la Institución de Educación Universitaria redefinida, reformulada en función e identificada con la sociedad y puesta a su asistencia, servicios, utilidad e intereses para el beneficio de las mayorías.

Partiendo de los supuestos anteriores, las Universidades Venezolanas deben abocarse a lograr conseguir el redimensionarse, examinar sus objetivos, ponderar sus propósitos, reconstruir su finalidad, resurgir en tiempos de cambios, de implosión social; en un movimiento educativo colectivo comunitario popular, con la característica particular de estar ávido, propenso e inclinado a la búsqueda y consecución efectiva de soluciones prácticas, eficaces, eficientes, perdurables que permitan satisfacer, resolver con resultados positivos-productivos las necesidades y problemas de la sociedad. 
Frente a este escenario, es propicio señalar que para alcanzar, coadyuvar y facilitar en el proceso de encontrar las soluciones es indicado enfatizar, recalcar que se debe contar con la garantía de la participación ciudadana democrática, en igualdad de condiciones, de forma libre, pluralista, equitativa y protagónica de todos los actores, sectores sociales y factores productivos involucrados en el proceso, en una constante, permanente e inalterable búsqueda de la verdad.

Para cumplir tal procedimiento, la Organización de las Naciones Unidas para la Educación, la Ciencia y la Cultura (UNESCO, 1998): expone que se debe tomar en cuenta la diversidad, diferencias, heterogeneidad, variedad, de los espacios universitarios para el debate de los planteamientos, la confrontación de las ideas, la crítica con sólidos argumentos, el disenso de los pensamientos, la discusión de altura, la reflexión académica-investigativa, para emprender el alcance, obtención de la transformación de la sociedad, en una sociedad con calidad humana, competitiva, ecuánime, estable, más justa, altamente preparada y solidaria, con los preceptos de una Educación de avanzada, dinámica, moderna, renovadora, productiva, sustentable e inventiva.

Dentro de esta perspectiva, la formación de un moderno Profesional Universitario comprende, conlleva, significa e implica en paralelo la formación de un nuevo, actual e innovador Profesor Universitario, por ello es importante resaltar que este inédito Profesor formado, capacitado, preparado e instruido en la formalidad de un sistema educativo normado por la doctrina, principios e ideales del trabajo colaborativo, participativo, interactivo, creativo, crítico y transdisciplinario, el cual debe atenuar, debilitar, disminuir las limitaciones de los roles tradicionales en los procesos enseñanza-aprendizaje, por consiguiente, como producto debe estimular el desarrollo de conciencias críticas, ayudar a la creación de conocimiento nuevo, propiciar la generación de atrayentes disciplinas, incipientes ciencias, interesantes saberes y alterar, 
Instituto Internacional de Investigación y Desarrollo Tecnológico Educativo INDTEC, C.A.

DOI: https://doi.org/10.29394/Scientific.issn.2542-2987.2021.6.21.19.356-375

OAI-PMH: http://www.indteca.com/ojs/index.php/Revista Scientific/oai

Ensayo Original / Original Essay

cambiar, revolucionar la aparente quietud, placidez, sosiego, tranquilidad e inacción del aula de clases.

\subsection{Formación del Personal Académico versus Realidad Universitaria}

El tópico de las políticas de la formación y preparación del Personal Profesoral en el ámbito académico universitario en virtud de la significación, e importancia que detenta para la consecución del éxito de los propósitos y finalidades de la organización educativa universitaria, es de atención preponderante en el momento que se intenta el valorar desde el punto de vista analítico, metódico y determinar la ubicación que respectivamente una Institución de Educación Universitaria posee en lo referente a calidad, congruencia, naturaleza y trascendencia en el contexto regional, nacional e internacional.

A este respecto está explícita y tácitamente entendido, establecido e instaurado en diversos escenarios académicos el fundamento que sin un Personal Docente con admirables, extraordinarios y respetables conocimientos, elevados niveles de competencias, credenciales de robusta formación, idónea preparación, apto he instruido formidablemente, calificado para dar contestaciones bien analizadas, con sólidos argumentos, juicios críticos y soluciones globales a las demandas, preguntas y problemas que le propone el contemporáneo pensamiento epistemológico, filosófico, las Universidades no tendrían objeto, ni razón alguna de existir.

Al mismo tiempo, el Profesor Universitario debe transitar los adelantos y la evolución de los campos científico, tecnológico, informático, telemático, e inteligencia artificial, el progreso de los entornos ambientales, culturales, económicos, sociales, de vincularse con afinidad, socializar con creatividad, e interactuar de forma inventiva, con inteligencia crítica, cuestionar con consistentes razonamientos, analizar y examinar las relaciones presentes en las diversas comunidades, la colectividad y los grupos sociales que conforman 
la sociedad en general, en concomitancia con el Estado, el Gobierno Nacional, los sectores Laborales, Empresariales y Productivos tanto Públicos como Privados.

En fin, con base en lo expuesto las Instituciones de Educación Universitaria podrían conseguir la prerrogativa de ser y permitirse el hecho de coexistir solo de forma estructural, pero adoleciendo de su esencia, finalidad, naturaleza y sin tener el carácter medular, ni la cualidad sustancial de su existencia "per se". Atendiendo a estas consideraciones, las Instituciones de Educación Universitaria pasarán a ser una simple entidad educativa organizacional indiferente, impasible, pasiva, despreocupada e inerte ante la sociedad, en lugar de ser una entidad fundamental, creadora, decisiva, dinámica, eficiente, proactiva, productiva e innovadora para construir, generar y proporcionar los avances, cambios, el crecimiento, desarrollo y trasformaciones que la misma sociedad actual demanda, exige, reclama, requiere de las Instituciones de Educación Universitaria.

Es necesario sostener que las Instituciones Universitarias Venezolanas están en el deber de formar a un profesional que genere altos niveles de desempeño en los ámbitos: académico, profesional, laboral y científico, por el contrario, la realidad del hoy en día es que la labor de los Profesores Universitarios se enmarca, minimiza, limita y reduce en atender a los estudiantes para solo impartir clases, sin plasmar proyectos por competencias, realidades como planes de acción que incentiven y produzcan conocimientos para expresar, debatir y analizar en los diversos espacios del intercambio de saberes que permita divulgar los adelantos, avances y progreso de la investigación científica, al igual que, intercambiar las experiencias en las diversas áreas del conocimiento.

Para ello, Tobón (2013), citado por Gonzalez-Guacaneme (2017): amplia la visión considerando el enfoque socioformativo que "busca formar la competencia, donde se "aplica el pensamiento complejo en la comprensión y 
abordaje de las competencias en la educación, para promover la formación integral, teniendo en cuenta los desarrollos conceptuales en este campo" (pág. 1); desde el pensamiento complejo-aprendizaje colaborativo.

Tales efectos hacen que las Instituciones de Educación Universitaria cuentan en minusvalía con una base de datos, capaz de organizar además de registrar todos y cada uno de la data referente al talento humano profesoral de las diversas Instituciones de Educación Universitaria del país, que pueda generar un cúmulo de competitivos profesionales capaces de elevar la excelencia académica como participar activamente en el desarrollo de planes científicos-educativos-económicos-socio-productivos para la Nación.

En ese mismo orden de ideas, para Marqués (2000), citado por Mosquera (2012): la tecnología educativa ha jugado un papel muy importante en el diseño de ambientes de aprendizajes en medios electrónicos (Internet), con el propósito de confrontar diversas "situaciones instruccionales que permitan alcanzar los objetivos propuestos por determinados programas educativos, apoyando la función del análisis y evaluación a la luz del contexto de su aplicación" (pág. 32). La idea es diseñar, producir e implementar técnicas y materiales con el apoyo de la tecnología para promover sistemas educativos efectivos.

Asimismo, es considerable y esencial hacer el esfuerzo en calidad de la enseñanza como punto focal en las Instituciones de Educación Universitaria; incorporar en lo posible los objetos de aprendizaje, que no son más que cualquier tipo de recurso digital que debe ser utilizado como parte del aprendizaje E-learning con la finalidad de apoyar el proceso de aprendizaje en los espacios de clase universitario. La educación de ahora debe asumir nuevos roles, donde cada vez se rompen con los paradigmas y percepciones obtusas fragmentando los resquemores, dudas y falsas percepciones de la Web, por tanto, la Educación debe hacer de la tecnología su principal aliada para no ser erosionada por los avasallantes avances tecnológicos. 
No obstante, es bueno señalar la experiencia en las Instituciones de Educación Universitaria que forman Educadores que practican y lideran su proceso de formación en varias fases, un completo proyecto que enmarca la formación docente a nivel universitario, por cuanto, el día a día implica impartir a sus estudiantes conocimientos, saberes, destrezas, habilidades y pericias de una profesión, y eso permite, según Inciarte y Canquiz (2008): una formación integral permanente, que produzca una proactividad docente, para así, poder asimilar los cambios producto del desarrollo científico, tecnológico y humanístico, con el fiel propósito de mantenerse actualizados con los nuevos enfoques, teorías y construcción de nuevos elementos epistemológicosfilosóficos que se generan y condensan de forma íntegra al Profesor Universitario capacitado, activo para la identificación, aportes como planteamientos a posibles soluciones en los espacios que se requiera.

En contraposición a lo antes expuesto, las Instituciones de Educación Universitaria van hacia un futuro no muy lejano, a la desmasificación universitaria por los disímiles compromisos y gastos que la administración central debe desembolsar, la tecnología e informática que ahora permite trabajar desde cualquier parte con recursos y habilidades tecno-aula propias del Profesor Universitario como Tablet, PC, laptop, teléfonos inteligentes, aunado, combinado e integrado por un amplio e inmenso número de páginas, blog, portales web con aulas virtuales que utilizan e implementan sistemas de interacción asociativa de interrelación de saberes Profesor-Estudiante.

De este modo, diseñan un nuevo currículo con la Modalidad de Educación a Distancia posible para muchos, aun cuando los organismos institucionales deben sugerir, procurar, invertir y proveer la dotación de equipos, asimismo de aulas virtuales de fácil acceso como un novedoso sistema único de interconexión para poder tener planes, currículo, didáctica, estrategias, sistemas, técnicas e instrumentos de evaluación fundamentados en el apoyo de la tecnología e informática. 
Instituto Internacional de Investigación y Desarrollo Tecnológico Educativo INDTEC, C.A.

DOI: https://doi.org/10.29394/Scientific.issn.2542-2987.2021.6.21.19.356-375

OAI-PMH: http://www.indteca.com/ojs/index.php/Revista Scientific/oai

Ensayo Original / Original Essay

\subsection{Visión Transformadora Universitaria}

De acuerdo con su naturaleza, las Instituciones de Educación Universitaria requieren en el presente de una mirada cuidadosa, juiciosa y reflexiva a su rol congénito, inherente, esencial e innato de compromiso y corresponsabilidad con el país, que exhibe, presenta e infunde en los actuales momentos, conllevando a generar una importante, significativa revisión hacia el núcleo central e intrínseco de su estructura organizacional.

En este sentido, es pertinente resaltar, que es fundamental, medular, substancial e imprescindible manifestar la exigencia a las Instituciones de Educación Universitaria de una nueva Visión Transformadora Universitaria dirigido a:

1. Debatir entre las Universidades: las necesidades de formación académica, las nuevas formas de comunicación, socialización e interacción, los sistemas y tendencias de la evaluación, uso de las aplicaciones, dispositivos, equipos y herramientas tecnológicas, Tecnologías de la Información y la Comunicación (TIC) que permitan la utilización de la tecnología e informática interactiva favorable al producto-valor del conocimiento, visiones sobre Desarrollo y Educación.

2. Concebir, visualizar la creación, construcción, divulgación y ejecución de conocimientos por medio de un Plan Nacional cuyo objetivo e intención sea desplegar y llevar a cabo en las Instituciones de Educación Universitaria un verdadero Programa de Transformación Universitaria, con vista a analizar, debatir, deliberar y difundir sus alcances, efectos, logros, productos, resultados en Congresos internos, locales, nacionales y regionales sobre Transformación Universitaria, incluso gestionando la participación de organismos a nivel mundial: Banco Mundial, Espacio Europeo de Educación Superior (EEES), Organización para la Cooperación y el Desarrollo Económicos (OCDE), Organización de Estados Iberoamericanos para la Educación, la Ciencia y la Cultura (OEI), Organización de las Naciones Unidas para la Educación, la 
Ciencia y la Cultura (UNESCO), el Programa de las Naciones Unidas para el Desarrollo (PNUD).

3. Expandir la misión de la Institución de Educación Universitaria, para concatenar espacios de debate, mesas de trabajo, intercambios universitarios, que confronten las realidades con la conexión-enlace-vínculo de las funciones docentes: academia, investigación, extensión y comunidad que reavive la participación universitaria-ciudadana, donde haya coincidencias, directrices, nuevos enfoques y crear ideas de posibles soluciones aplicables, legítimas y válidas con la realidad del país.

4. Enlazar y establecer alianzas, convenios, colaboraciones, sociedades con otras Instituciones de Educación Universitaria de carácter Público y Privado para promover acuerdos, intercambios, generar modelos prácticos de formación académica que impulse el accionar profesoral interactuando con la realidad como ente activo-participativo.

5. Se sugiere, proponer incorporar la actualización, formación y preparación permanente del Profesor como aspectos de evaluación docente en su desempeño educativo universitario que cause, genere y adecúe estímulos e incentivos en los docentes mejores formados académicamente.

6. Las Instituciones de Educación Universitaria deben crear, instalar e instituir espacios y plataformas tecnológicas para el desarrollo vanguardista profesoral en conocimiento para el desarrollo tecnológico-informativo-moderno que aplique diseños, proyectos, programas, modelos, capacitación y asesoría como planes de acción propios a las necesidades del Profesor Universitario en los espacios educativos virtuales.

7. Generar y renovar espacios abiertos con las asociaciones empresariales privadas, los organismos públicos municipales, regionales, y nacionales, así como con las entidades e instituciones dedicadas al fortalecimiento del desarrollo humano para actualizar, rediseñar, modernizar y revalorizar los pensum de estudio, para así revisar quién forman, a quién y con 
qué objetivos, finalidades, variables o parámetros congruentes de las necesidades reales del mercado.

8. Promover la organización e instauración formal de eventos, intercambios, Congresos, Seminarios y Talleres de alto perfil e índole académicos-científicos-didácticos-instruccionales anuales para el análisis, consideración, compartir, disertación, divulgación y estudio de experiencias educativas universitarias desde diversas áreas, diferentes disciplinas del saber, presentar y demostrar los logros significativos de las actividades, del quehacer, y del trabajo universitario desde cada proceso de interacción profesor-estudiante.

9. Contar con el establecimiento formal y legal de Convenios Gubernamentales e Interinstitucionales, que determinen e instituyan las fuentes de financiamiento y otorguen los respectivos recursos económicos dirigidos a estimular, financiar, promover e incentivar la constante actualización docente, perenne formación profesoral, continua preparación académica, prácticas virtuales, desarrollo de proyectos ambiciosos de investigación, participación en eventos nacionales e internacionales.

10. Disponer la creación de una amplia y completa Base de Datos Informativos Digitalizados-Electrónicos de los Cuerpos Profesorales de las Instituciones de Educación Universitaria que conlleve al diseño, construcción y elaboración de un Registro Estadístico muy ambicioso donde se describan, detallen minuciosamente y resguarden la cantidad, rotación, porcentajes relacionados a la formación académica, para determinar las fortalezas y oportunidades con que cuentan las Instituciones de Educación Universitaria.

\section{Conclusiones}

A modo de concluir, se plantea que debe existir una nueva forma de concebir, planear y proyectar las diversas situaciones del entorno Educativo, en especial, el del ecosistema Universitario, al mismo corresponde centrarse 
en generar, suscitar e innovar una visión amplia conforme a las necesidades y sobre todo a las realidades que cohabitan con el contexto, resaltando que el rol de la formación académica toma un valor realmente incalculable, indefinido considerando una revisión del qué se hace, cómo se hace; creando una generalización encaminada a fortalecer y con el propósito de beneficiar a todos en comunicación, mediación e interposición de respuestas acordes a tantas problemáticas de la actualidad.

Todos los elementos pragmáticos tratados en este ensayo, tienen un fin u objetivo común: un cambio, una nueva forma de pensamiento que derive en la adaptación, regulación como creación de conocimientos posibles, reales, aplicables y propios para atender, resolver y satisfacer de forma perdurable, positivamente las situaciones problemáticas en la sociedad. Por ello, la Formación Académica de hoy en adelante, debe centrarse en preparar un ser crítico, capaz de producir y originar un proceso convivalente, que envuelve todas las actividades humanas con su formación profesional encauzada, enfocada en un Proyecto Educativo flexible, cambiante, adaptable, transformado y contemporáneo.

Cabe destacar que es ahora cuando por medio de todas las experiencias como vivencias confrontadas proviene el cambio y el llamado a una verdadera, eficaz y profunda Transformación Universitaria capaz de formar a los docentes en el día a día del salón de clase y siendo los Profesores los impulsadores de una academia que busque construir, producir, contrastar, socializar e internalizar ese nuevo conocimiento teórico - práctico con pertinencia, congruencia y compromiso social, de calidad, interactuante con los procesos del entorno a todo nivel, en procura de seguir reconociendo el talento humano universitario brillante y presente en el quehacer educativo.

Por todo lo mencionado con anterioridad, es imperante que las Instituciones de Educación Universitaria deben crear e implementar políticas educativas en concordancia con los planes educativos de los organismos 
nacionales o internacionales en materia de Educación Universitaria, dado que representan y manejan datos macro de la realidad contextual con base en experiencias, casos de análisis, planes de acción y lineamientos como estrategias, con el propósito de contribuir a la Visión Transformadora Universitaria. Adicionalmente, tomando en cuenta el despliegue administrativo-jurídico-legal vigente en nuestro país que garantice el buen desempeño, observancia y alcance de los objetivos propuestos en el ámbito universitario.

También, es fundamental, primordial e inclusivo que dentro, a lo interno de las Instituciones de Educación Universitaria, el incentivar con la puesta en marcha de espacios para el análisis, la discusión y el debate académico profesoral donde se construya los planes de acción y los programas curriculares que ambicionen, procuren e intenten actualizar, formar, preparar al cuerpo profesoral, en función de concebir la excelencia, calidad y cualidad en Educación Universitaria.

Finalmente, el llamado a las Instituciones de Educación Universitaria en favor de beneficiar su cuerpo profesoral; es crear, constituir y establecer de forma continua e inseparable desde sus inicios en el espacio académico, una formación docente permanente a través de cursos, congresos, seminarios, conferencias, talleres, intercambios con otras universidades, rescatar los planes de financiamiento y estímulo con estudios en el escenario nacional y en el extranjero en prestigiosas universidades como parte del crecimiento del talento humano profesoral con reconocida trayectoria académica, con la meta de mejorar, formar, actualizar, ilustrar, adiestrar, capacitar, e instruir al Profesor Universitario, considerando sus amplias funciones académicas: docencia, investigación y extensión para así lograr, el desarrollo del valor humano profesoral en favor de las necesidades como posibles soluciones a las problemáticas reales del contexto país. 
Ensayo Original / Original Essay

\section{Referencias}

Cabero, J. (coord.). (2007). Tecnología Educativa. ISBN: 84-481-5613-7. España: McGraw-Hill.

Escamilla, J. (2012). Selección y uso de la tecnología educativa. México: Editorial Trillas.

García, C. (1998). Situación y principales dinámicas de transformación de la educación superior en América Latina. 3ra Edición. Caracas, Venezuela: UNESCO-IESALC.

Gonzalez-Guacaneme, G. (2017). Las competencias y el enfoque socioformativo: Una propuesta para transformar la educación. En Tobón, S. (coord.). Foro de Evaluación Socioformativa. (págs. 1-10). México: Centro Universitario MARCO.

Inciarte, A., \& Canquiz, L. (2008). Formación Integral desde el Enfoque por Competencias. Colección textos universitarios. Primera edición, ISBN: 978-980-7140-55-3. Maracaibo, Venezuela: Formación Integral desde el enfoque por competencias.

Mosquera, J. (2012). Integración de herramientas tecnológicas al proceso de enseñanza-aprendizaje para favorecer la competencia lectora en los niños del nivel preescolar de la Institución Educativa el Tres. Trabajo de grado. Bucaramanga, Colombia: Universidad Autónoma de Bucaramanga. Recuperado de:

http://hdl.handle.net/20.500.12749/2920

UNESCO (1998). Autonomía, responsabilidad social y libertad académica. Debate temático. La Educación Superior en el siglo XXI: visión y acción. París, Francia: Organización de las Naciones Unidas para la Educación, la Ciencia y la Cultura.

Yarzábal, L. (1999). Consenso para el Cambio en la Educación Superior. Colección Respuestas N. 9. Caracas, Venezuela: IESALC/UNESCO. 


\section{Ensayo Original / Original Essay}

\section{Maribel Josefina Díaz Castellanos \\ e-mail: maribdiazcastellanos@gmail.com}

Nacida en el Municipio Lagunillas, estado Zulia,

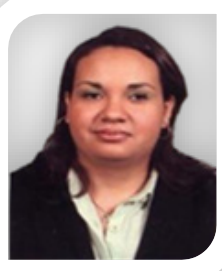
Venezuela el 16 de junio del año 1981. Licenciada en Administración egresada de la Universidad del Zulia (LUZ), Maracaibo, estado Zulia, República Bolivariana de Venezuela; cuento con estudios culminados de Componente Docente y Maestría en Gerencia Financiera de la Universidad donde ejerzo y pertenezco al cuerpo Profesoral, Universidad Nacional Experimental "Rafael María Baralt" (UNERMB); siendo actualmente Profesora en la categoría de Asociado con dedicación a Tiempo Completo; adscrita al Programa Ingeniería y Tecnología (PIT) de la Universidad Nacional Experimental "Rafael María Baralt"; con más de 15 años continuos de experiencia laboral profesional en el área de Lenguaje y Expresión; impartiendo como titular de las Asignaturas de Técnicas de Estudio, entre otras materias del Pensum de Estudios del Programa Ingeniería y Tecnología (PIT); además de ejercer Cargos Gerenciales como Coordinadora de Enlace Comunitario del Servicio Comunitario del Programa Ingeniería y Tecnología (PIT) de la UNERMB; en el presente, culminé la escolaridad del Programa de Postgrado de la misma institución.

El contenido de este manuscrito se difunde bajo una Licencia de Creative Commons ReconocimientoNoComercial-Compartirlgual 4.0 Internacional 